\title{
Opportunity rover localization and topographic mapping at the landing site of Meridiani Planum, Mars
}

\author{
Rongxing Li, ${ }^{1}$ Raymond E. Arvidson, ${ }^{2}$ Kaichang Di, ${ }^{1}$ Matt Golombek, ${ }^{3}$ Joe Guinn, ${ }^{3}$ \\ Andrew Johnson, ${ }^{3}$ Mark Maimone, ${ }^{3}$ Larry H. Matthies, ${ }^{3}$ Mike Malin, ${ }^{4}$ Tim Parker, ${ }^{3}$ \\ Steven W. Squyres, ${ }^{5}$ and Wesley A. Watters ${ }^{6}$ \\ Received 14 June 2006; revised 14 November 2006; accepted 8 December 2006; published 14 February 2007.
}

[1] This paper presents the results of Mars topographic mapping and lander and rover localization for the Opportunity rover at Meridiani Planum during the Mars Exploration Rover (MER) 2003 mission. By Sol 458, the Opportunity rover traversed a distance of $5.20 \mathrm{~km}$. We localized the lander using two-way Doppler radio positioning and cartographic triangulation of craters visible in both orbital and ground images. Additional high-resolution orbital images were taken to verify the determined lander position. Visual odometry and bundle adjustment techniques were applied to overcome wheel slippages, azimuthal angle drift, and other navigation errors (as large as $21 \%$ within Eagle crater). In addition, orbit-to-ground image-based adjustment was applied to correct rover location errors where bundle adjustment was not applicable. We generated timely topographic products, including orthoimages, digital terrain models (DTMs), threedimensional (3-D) crater models, and rover traverse maps. In particular, detailed 3-D terrain models of major features, such as Endurance crater, have been generated using multisite panoramic stereo images based on bundle adjustment and wide baseline stereo technique.

Citation: Li, R., et al. (2007), Opportunity rover localization and topographic mapping at the landing site of Meridiani Planum, Mars, J. Geophys. Res., 112, E02S90, doi:10.1029/2006JE002776.

\section{Introduction}

[2] After a successful landing on January 25, 2004 at Meridiani Planum, as of Sol 458, the Opportunity rover of the Mars Exploration Rover (MER) 2003 mission traveled a distance of $5.20 \mathrm{~km}$ (actual distance traveled) from the lander (Figure 1). The rover started with detailed science investigations within Eagle crater (22 $\mathrm{m}$ diameter) for the first 56 sols where the spacecraft landed. After a traverse of $685.4 \mathrm{~m}$ and a short stop at Fram crater (8 m diameter), Opportunity rover reached a large crater of $156 \mathrm{~m}$ diameter, Endurance crater, on Sol 95 where the rover traversed along the rim before descending into the crater to perform a 318 sol extensive exploration. On Sol 325, Opportunity arrived at the place where the heatshield of the landing craft crashed and inspected the site for engineering analysis.

\footnotetext{
${ }^{1}$ Department of Civil and Environmental Engineering and Geodetic Science, Ohio State University, Columbus, Ohio, USA.

${ }^{2}$ Department of Earth and Planetary Sciences, Washington University, St. Louis, Missouri, USA.

${ }^{3}$ Jet Propulsion Laboratory, California Institute of Technology, Pasadena, California, USA.

${ }^{4}$ Malin Space Science Systems, San Diego, California, USA.

${ }^{5}$ Department of Astronomy, Cornell University, Ithaca, New York, USA.

${ }^{6}$ Department of Earth, Atmospheric and Planetary Sciences, Massachusetts Institute of Technology, Cambridge, Massachusetts, USA.

Copyright 2007 by the American Geophysical Union. 0148-0227/07/2006JE002776
}

Farther down south, $1083 \mathrm{~m}$ from Endurance crater, the rover traveled among three closely located small craters, Investigator, Géographe and Naturaliste. By Sol 405, Vostok crater (40 m diameter) was behind the track and the rover traversed $3343.6 \mathrm{~m}$ in total and was on the way to approaching Erebus crater (320 m diameter).

[3] A typical study of a ground feature, such as a rock, would start with remote-sensing observations using the Pancam [Bell et al., 2006] and Miniature Thermal Emission Spectrometer (Mini-TES) (P. Christensen et al., Mini-TES overview, submitted to Journal of Geophysical Research, 2006) systems to identify key targets for detailed study. The rover was then commanded to traverse to the targets, and fine-scale positioning was used to place the vehicle in locations where the instrument deployment devices (IDD) could be used to place the Mössbauer Spectrometer (MB), Alpha Particle X-Ray Spectrometer (APXS), Microscopic Imager (MI), and/or Rock Abrasion Tool (RAT) tools onto surfaces for acquisition of in situ observations. All of these operations required three-dimensional (3-D) terrain information and accurate tracking of the rover's location to ensure efficient drives to the targets and to acquire desired science observations [Arvidson et al., 2004; Biesiadecki et al., 2005a]. Furthermore, precision localization of the traverse and conversion to the Mars inertial and MBF (Mars bodyfixed) coordinate systems [ $\mathrm{Li}$ et al., 2004a] were necessary for long-term traverse planning on orbital maps and correlation analysis of orbital and rover-based observations. 


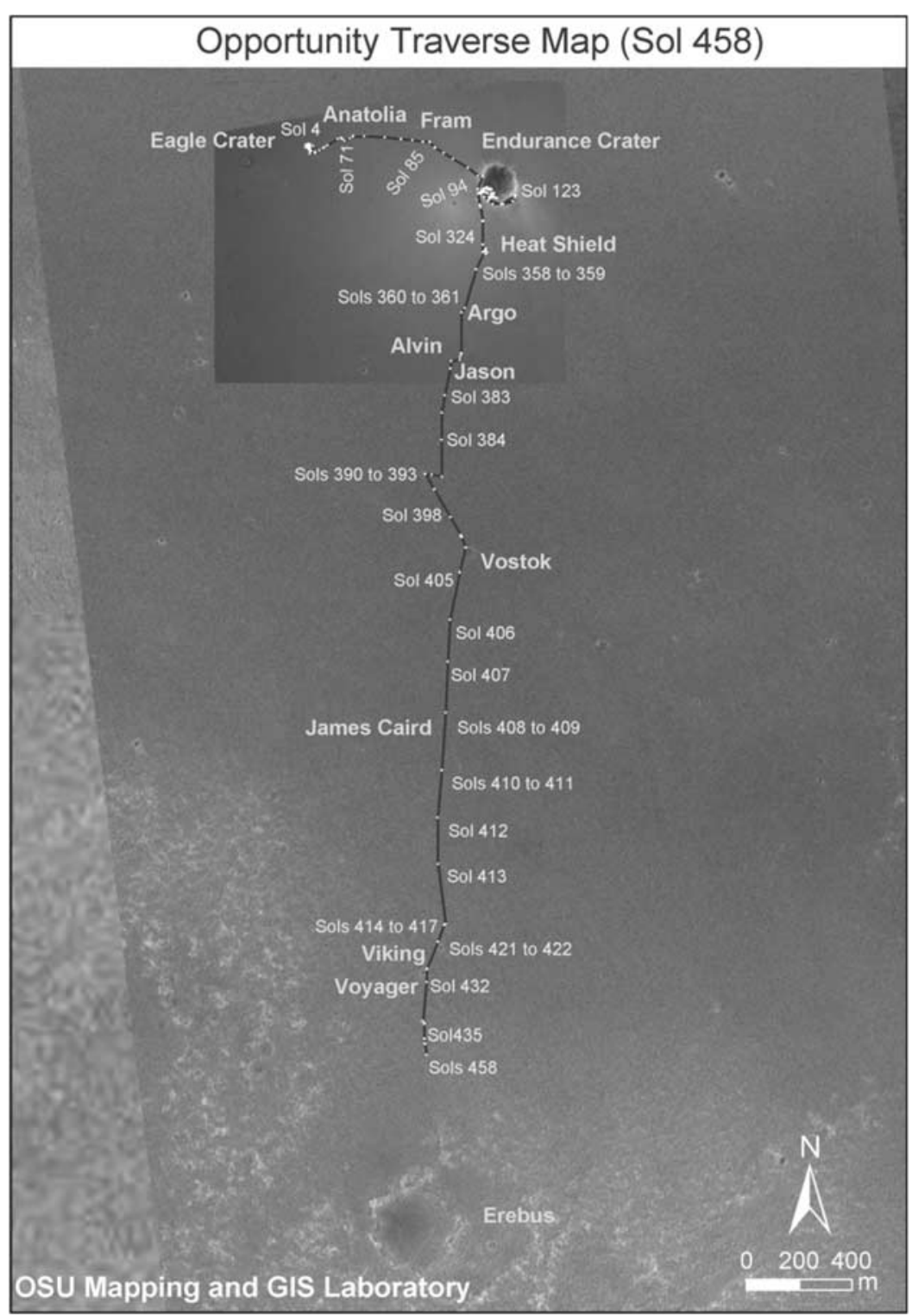

Figure 1. Opportunity rover traverse up to Sol 458 displayed on the base map generated using DIMES and MOC NA images.

[4] Similar to the Gusev landing site [Li et al., 2006], prelanding orbital images including those acquired by the Viking vidicon camera, the Odyssey Thermal Emission Imaging System (THEMIS), and the Mars Global Surveyor (MGS) Mars Orbiter Camera (MOC) were available. Most topographic products derived from the orbital images, such as the U.S. Geological Survey (USGS) Mars Digital Image Mosaic (MDIM 2.1), are referenced to the MBF reference system. The best accuracy achieved by such orbital dataderived maps is about $100 \mathrm{~m}$ [Li et al., 2004a]. Post landing, MGS took additional ROTO (roll-only targeted observation) images of the Opportunity landing site with a resolution of $1 \mathrm{~m}$ [Malin, 2004]. Stereo mapping was performed by Athena team members at the U.S. Geological Survey using MOC NA images to produce DTMs of representative terrains in candidate MER landing sites before the landing [Kirk et al., 2003]. Although the high resolution of the MOC NA images (approximately $3 \mathrm{~m}$ for the majority of images) can result in high quality DTMs $(10 \mathrm{~m}$ grid spacing), a MOC NA stereo image pair generally consists of one nadir and one oblique image, or two suitable oblique images covering the same overlapping area which is small (maximum swath width of $3 \mathrm{~km}$ ). Therefore overlapping images suitable for stereo analysis are rare. Stereo MOC NA images did not exist at the Opportunity landing site before landing. A MOC NA stereo pair (images R14-00021 and R14-01689) of the planned traverse was acquired in February 2004. However, the terrain in this region is generally flat and does not provide sufficient image texture for software to generate quality DTMs and orthoimages.

[5] During descent of the lander, three DIMES (Descent Image Motion Estimation System) sequential images were taken at about $1000 \mathrm{~m}$ with a ground resolution of about $1 \mathrm{~m}$ for the purpose of precision landing. The images were employed on board for autonomous estimation of horizontal velocity and spacecraft navigation in the presence of 


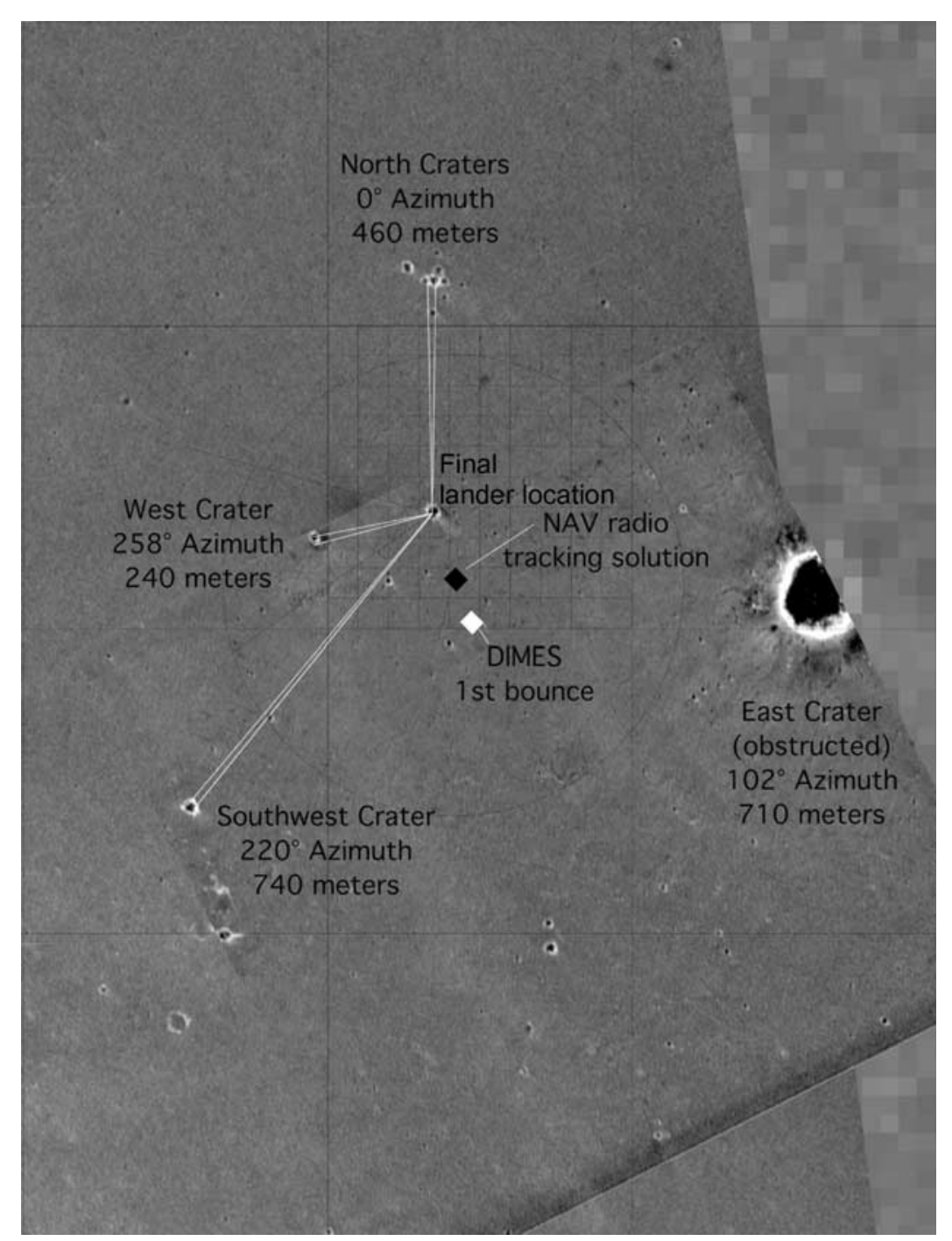

Figure 2. Opportunity lander location: high-resolution DIMES and MOC mosaic of the area around the Opportunity lander. Black square shows the location of the navigation solution. White square shows the first bounce point from the DIMES EDL reconstruction. Lines converging at the final lander location show azimuths to crater rims clearly visible from the Opportunity lander.

Martian winds. The same images were also used for trajectory reconstruction of the spacecraft EDL (entering, descending, and landing) process. Consequently, they were a very important data set for lander localization.

[6] This paper focuses on the results of Mars topographic mapping and lander and rover localization for the Opportunity rover at Meridiani Planum through Sol 458 during the MER 2003 mission. We localized the lander by an initial fix through two-way Doppler radio positioning technology and an improvement by a cartographic triangulation method using rover, MOC NA (narrow angle) and DIMES images. The post landing MOC ROTO images verified the determined lander position. Wheel odometry (as will be explained later in the text) provided some crude estimates of the traverse information, however, owing to wheel slippage, the covered ranges tended to be overestimated. Visual odometry(VO) and bundle adjustment(BA) techniques were applied to overcome wheel slippages, azimuthal angle drift and other navigation errors and to provide accurate rover traverse information and improved pointing data for topographic mapping. Within Eagle crater, BA was able to correct relative traverse errors as large as $21 \%$. Various topographic products were generated routinely. In particular, detailed 3-D terrain models of major features, such as Endurance crater, were generated using multisite panoramic stereo images based on bundle adjustment and wide baseline stereo imaging and matching techniques. A web-based landing-site GIS system was established at The Ohio State University (OSU) to update and disseminate localization and topographic information to support daily tactical and longterm strategic operations of the mission.

\section{Localization of Lander}

[7] Mars global reference and local reference systems used for the MER mission are described by Li et al. [2004a]. Particularly, the Mars inertial reference system and the Mars body-fixed reference system are the global reference systems, and the local reference systems include the rover frame, site frame, and landing site cartographic (LSC) 


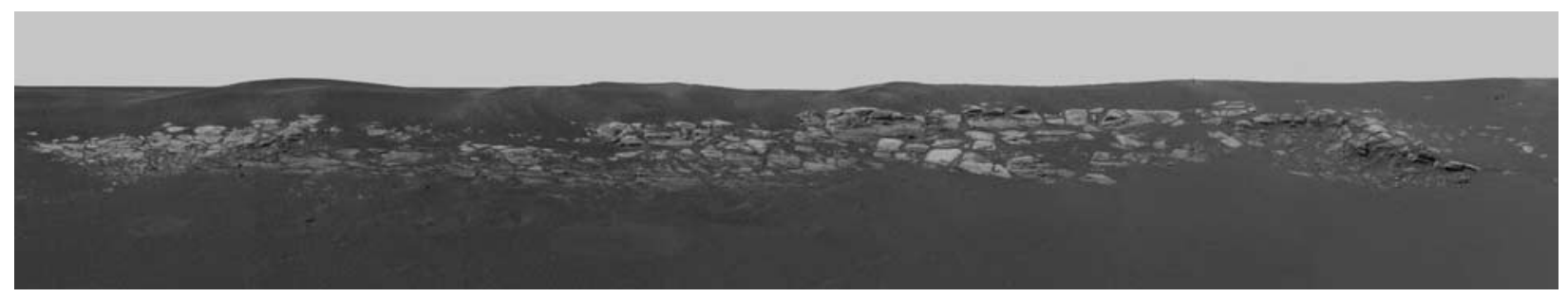

Figure 3. First Pancam panorama taken by the Opportunity rover at the landing site.

frame. They are implemented in the same way as at the Spirit landing site [Li et al., 2006]. The ground image-based topographic mapping and Opportunity rover localization is performed in the LSC reference frame, which is an eastnorth-up (X-Y-Z) right-handed coordinate system in meters with its origin at the lander.

[8] On January 25, 2004, the Opportunity rover successfully landed on Meridiani Planum. The location of the lander in the inertial reference system was determined by fitting direct-to-earth (DTE) two-way X-band Doppler radio transmissions and two passes of two-way Ultra High Frequency (UHF) Doppler transmissions between Opportunity and the Mars Odyssey orbiter. On the basis of analyses of these observations, the landing location is $1.9483^{\circ} \mathrm{S}$ (with an accuracy of about $10 \mathrm{~m}$ ) and $354.47417^{\circ} \mathrm{E}$ (with an accuracy of about $10 \mathrm{~cm}$ ), translated to International Astronomical Union (IAU) 2000 areocentric coordinates (see "NAV radio tracking solution" in Figure 2) [Guinn and Ely, 2004; Golombek and Parker, 2004; Arvidson et al., 2004]. The first Navcam panorama, taken on Sol 1, indicated that the spacecraft landed in a crater. The first Pancam panorama, completed on Sol 3, showed more details of the crater (Figure 3). However, a number of craters appeared in the surrounding area of the navigation solution in the MOC and DIMES images. So, the identification of the crater in which Opportunity had landed was not immediately clear. As one of the efforts to determine the exact crater of landing, a 3-D crater model was generated using a threetiered Pancam panorama. Crater parameters such as rim size and shape were measured from the crater model and compared with those from the DIMES images. The crater model was also employed to generate a simulated overhead view image (Figure 4). Although the simulated overhead view image does not perfectly match the crater in the DIMES image, the derived pattern provides the information that can be used to decide how to measure the size and shape on the DIMES image. The sizes and shapes of Eagle Crater measured in DIMES and simulated images are closer than those of three other craters in the vicinity [Li et al., 2004b].

[9] A reconstruction of the lander trajectory down to the surface and through bouncing to its final resting spot was performed using data collected during EDL (entry, descent, and landing). The reconstruction was generated by integrated angular rates and accelerations provided by the lander RIMU (rover inertial measurement unit) from an initial altitude and vertical velocity provided by the Radar Altimeter Subsystem (RAS) and a horizontal velocity provided by DIMES. This trajectory was in a local level, not a global reference frame, but the DIMES images could be rectified to the ground plane using this trajectory which allowed the trajectory to be compared to surface features. Once this was done (see Figure 5), the reconstructed trajectory was shown to intersect one of the craters to the northwest of the initial bounce location (Figure 3).

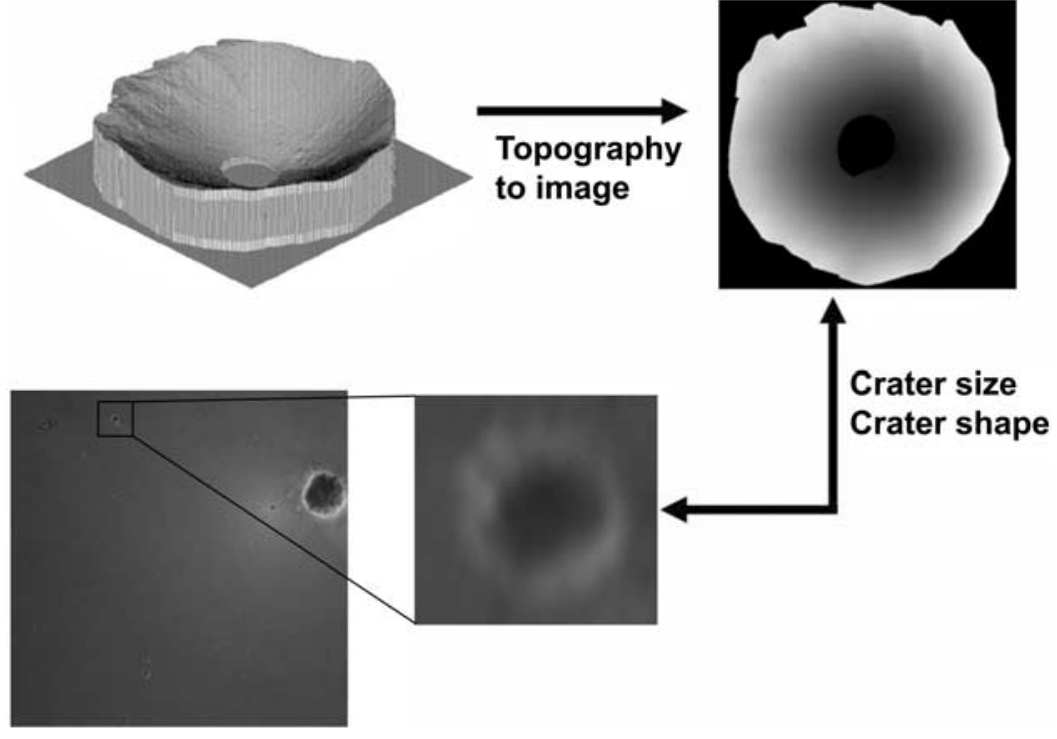

Figure 4. Three-dimensional crater model and derived image patterns were used for matching with the DIMES images for lander localization. 


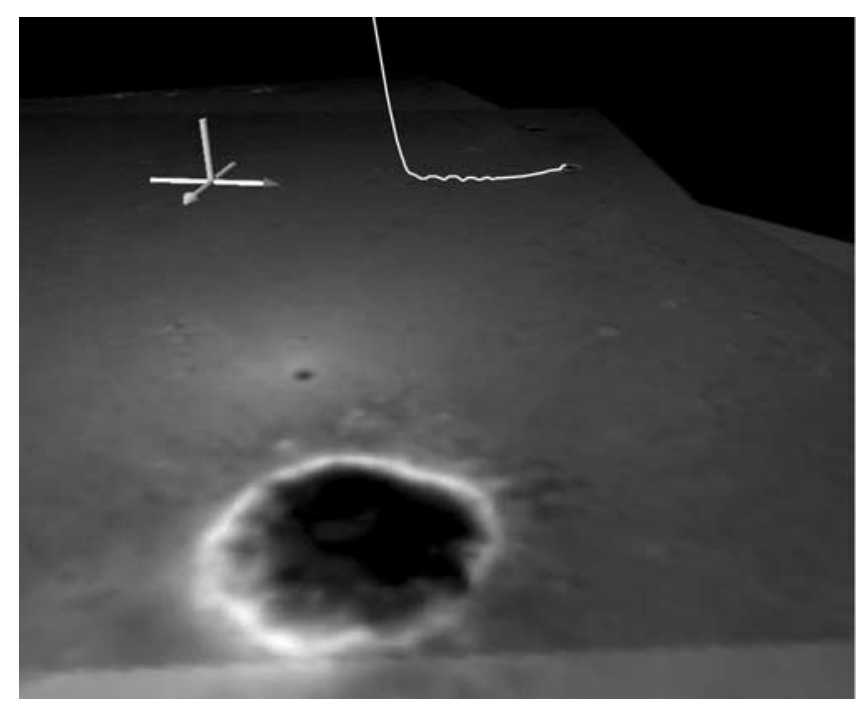

Figure 5. Reconstructed EDL trajectory showing final descending and bouncing superimposed on mosaic of 3 DIMES images and axes defining the local-level coordinate system used for trajectory reconstruction.

[10] Finally, the landing location was tied to the Mosaic of MOC NA and DIMES images to within an accuracy of about $10 \mathrm{~m}$, by triangulation to three craters observed in the far field through breaks in the local Eagle crater rim in Pancam images. These triangulation results, mapped to the cartographic network-derived MGS Mars Orbital Laser (MOLA) data, imply that the lander is located at $1.9462^{\circ} \mathrm{S}, 354.4734^{\circ} \mathrm{E}$ in IAU 2000 areocentric coordinates (see the crater in Figure 2 labeled "Final lander location" [Golombek and Parker, 2004; Parker et al., 2004]. The lander location was finalized on the basis of the above computational results using several localization technologies and was later nicknamed Eagle crater. In Figure 2, the mosaic of DIMES and MOC NA images of the area around the Opportunity lander was registered to the IAU 2000 areocentric coordinate frame and was used as the base map.

[11] Opportunity stopped on the plains in the Anatolia region for a software upload on Sols 75 to 78 , and its location $\left(1.9475^{\circ} \mathrm{S}, 354.4772^{\circ} \mathrm{E}\right)$ was determined by analysis of two passes of UHF two-way Doppler tracking. The location was also determined by image-based triangulation to common features, with resultant values of $1.9453^{\circ} \mathrm{S}$, $354.4766^{\circ} \mathrm{E}$. For both landing and software-upload locations, the Doppler-based location was displaced $135 \mathrm{~m}$ at an azimuth of $167^{\circ}$ clockwise from north from the location derived from image-based analyses (Figure 6). One displacement vector starts inside Eagle Crater and ends at the location pointed to by an arrow and labeled "inertial location, lander"; the other starts from a point close to Anatolia crater and ends the location pointed to by an arrow and labeled "inertial location, sol 78." This displacement is consistent with expected offset errors between inertially derived locations and positions estimated from the MOLA-based global cartographic network. These offsets provide a quantitative description of map errors expected for Mars in the equatorial regions when the current cartographic framework is used. The fact that the offset is so similar in azimuth and distance at the two locations suggests that both the inertial and cartographic localization techniques are

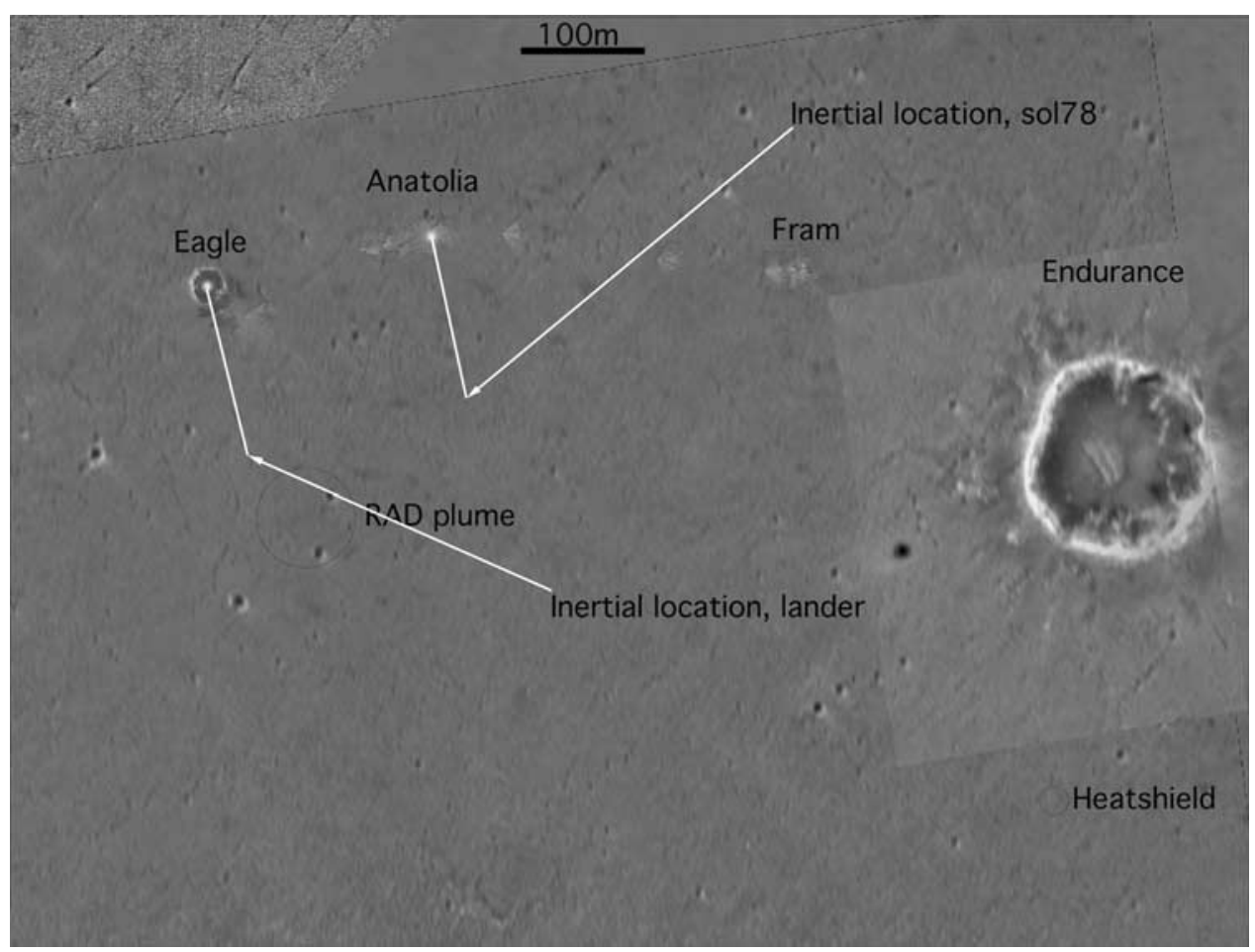

Figure 6. Rover locations determined by cartographic triangulation and radio science based on navigation techniques at the lander position and the software upload location as well as their offset vectors at the Meridiani Planum site. 
robust and accurate and no unaccounted-for random errors exist in either. The observed systematic offset is most likely an offset between the cartographic frame and the inertial frame and the observed offset is close to the 3 sigma estimate found in pre-landing maps [Golombek and Parker, 2004].

[12] The MOC NA orbiter imager aimed itself at the lander location and took a new, high-resolution (1 m) ROTO image at Eagle crater on Sol 13 by rolling the MGS spacecraft approximately $22.7^{\circ}$ off-nadir toward the west [Malin, 2004]. It is also the only orbital image in which the rover can be identified within the operation period addressed by this paper. The ROTO technique allows imaging off-nadir and at a higher resolution. Using the prelanding MOC NA images and the post-landing MOC ROTO image, the location of the lander was verified through changes in pixels (mainly contributed by the reflection of the solar panel). This orbital image is not stereo and therefore is not used for precision photogrammetric triangulation of the ground position. However, this information provided the important relative position of the rover with respect to the surrounding terrain features (e.g., Eagle crater). This position information from the MOC image was not introduced in the BA of the rover traverse.

\section{Rover Localization}

[13] MER rovers maintain an estimate of their 6 degreesof-freedom pose (3-D position and 3-D attitude) using several means. A Litton LN-200 Inertial Measurement Unit (IMU) provides solid state gyros for measuring attitude drift and accelerometers for finding the nadir vector; encoders on the wheel drive motors enable an approximate prediction of distance traveled; and pointable cameras enable autonomous detection and tracking of the Sun (for attitude updates) and nearby terrain features (for position updates).

[14] Rover attitude is the transformation from the rover body frame (X forward, $\mathrm{Y}$ right, $\mathrm{Z}$ down) into local level frame (X north, $\mathrm{Y}$ east, $\mathrm{Z}$ down, with the same origin as rover body frame [ $\mathrm{Li}$ et al., 2004a], and is typically represented using quaternion notation BqLL. This is a critical component of vehicle state. For instance, the high gain antenna must be capable of pointing at the Earth to within 2 degrees, 3 sigma. Vehicle attitude is assumed to stay fixed when neither the instrument arm nor the wheels are commanded to move. But whenever the rover is commanded to drive, and often when the arm is used, the IMU is turned on and the gyro readings are integrated to provide updates to the vehicle attitude. The accelerometers may also be used to check instantaneous tilt against a preset limit, but only the gyro readings contribute to the persistent vehicle attitude. Gyro drift will accumulate, so human controllers typically command the rover to reset its attitude after 10,000 seconds of IMU integration time (i.e., once every few weeks). At that time, BqLL pointing precision is improved by measuring the vector to the Sun (found by centroiding an image of the Sun in the Pancam) and combining it with the nadir vector measured by the accelerometers [Ali et al., 2005].

[15] Vehicle position is primarily estimated by combining the wheel encoder measurements from the two middle wheels with attitude changes measured by the gyros in the
IMU. This mode is known as "wheel odometry," because although the direction of motion is measured by an inertial sensor, the distance traveled is assumed to correspond directly to a function of the amount each middle wheel rotates. Although this estimation works well on flat ground, it fails to incorporate any information about wheel slip that commonly occurs in sandy, rocky or highly sloped terrain. When such situations are expected, human rover drivers will often command the rover to update its position knowledge during the drive by autonomously comparing images taken before and after each short drive step. In this mode, dozens of terrain features are autonomously located in Navcam stereo images and tracked in two dimensions and three dimensions, resulting in dozens of motion vectors that are robustly filtered to produce an accurate measurement of overall vehicle motion. This process of using images to improve upon the approximate vehicle position (which is only "predicted" by wheel odometry) is known as Visual Odometry [Cheng et al., 2006].

[16] Unfortunately, Visual Odometry processing can take several minutes per image pair, which greatly reduces the amount of distance that can be driven each sol, so it was only enabled in the more slippery or uncertain terrains such as the interior of Endurance crater and loose sandy areas between Endurance and Victoria craters. Although the total fraction of distance traveled in which it was used seems low (11\% of 5947 meters driven as of sol 555 [Biesiadecki and Maimone, 2006]), it enabled precision driving in the most dangerous (and most scientifically interesting) terrains. Earth-based tests with ground truth measured by total station have shown that Visual Odometry can produce position estimates accurate to within $2 \%$ of the total distance driven [Olson et al., 2003].

[17] Supported by manual slip prediction and VO technique, Opportunity rover drove very carefully in short segments to explore the outcrop within Eagle crater. Major difficulties were to overcome wheel slippage caused by crater wall slopes and loose soil. The drives performed on the plain after exiting the crater were longer, often exceeding $100 \mathrm{~m}$ per sol, because of the openness of Meridiani Planum and the less challenging terrain in the early part of the overall Opportunity traverse. In a typical long driving sol, the rover first performs a blind drive for a distance that can be visualized and accurately measured from Pancam or Navcam images (often over $20 \mathrm{~m}$ ), and then performs an autonomous drive (where hazard avoidance is performed in real time using Hazcam stereo images) until a time that is typically set by communication passes or energy constraints. Because autonomous driving is computationally intensive and hence slow, the autonomous portion of a drive often covers a shorter distance than the blind portion [Biesiadecki et al., 2005b]. Both blind and autonomous drive motion is typically based on simple primitive operations: straight line drives, curved arcs, or turns in place.

[18] Rover localization was conducted at several levels [Li et al., 2004a]. Within each sol cycle, the onboard IMUand wheel-odometry-based localization were always used with infrequent support from sun-finding techniques that improved the quality of the attitude. In cases where the rover experienced slippage caused by traversing on loose soil terrain or against steep slopes (particularly when in craters) the onboard VO was applied. VO is also used 

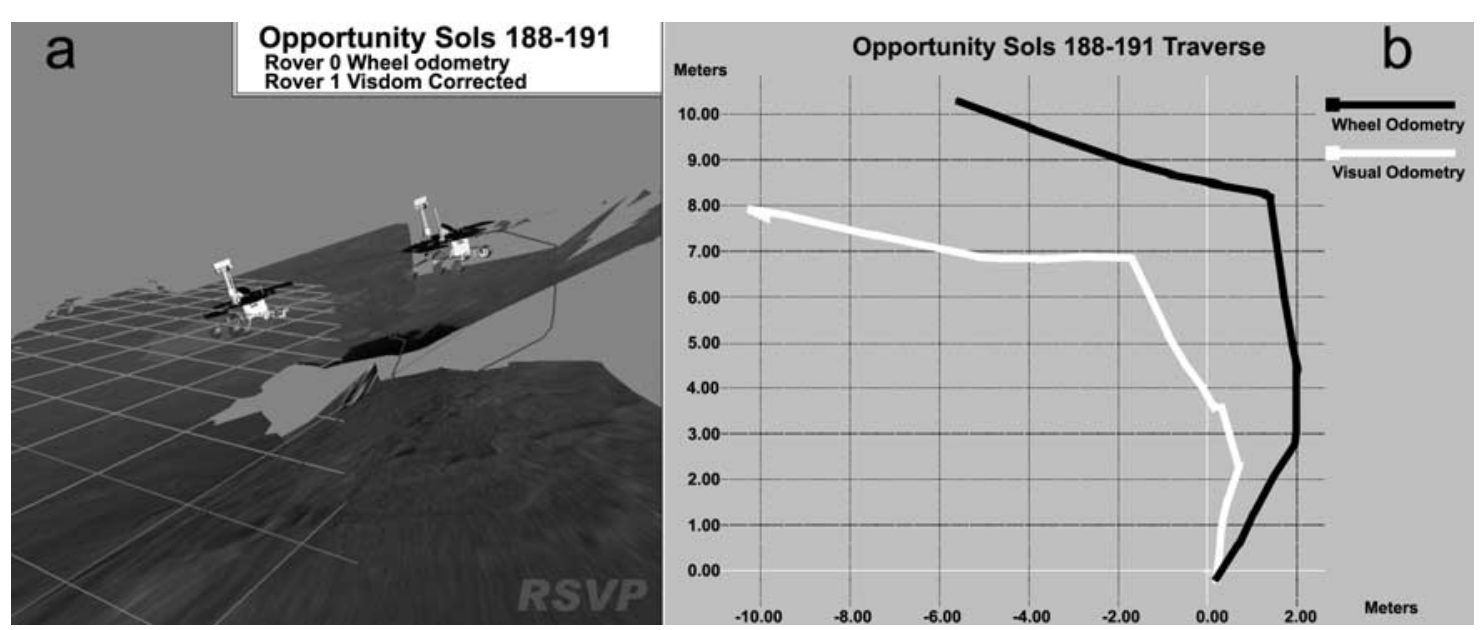

Figure 7. Two views of the impact of visual odometry on the rover's onboard position estimate. The rightmost path in both plots shows what path Opportunity would have estimated during a drive inside Endurance crater had it relied solely on its IMU and wheel encoders. The leftmost path shows the path as it was actually updated by visual odometry, which measured the actual downhill slip it encountered on the $17 \pm 2$ degree sandy slope. These estimates differ by over $5 \mathrm{~m}$ after only $17.6 \mathrm{~m}$ of actual driving.

whenever a highly accurate rover position is desirable, for example, to approach a science target. Details of VO are described by Olson et al. [2003], Cheng et al. [2005], and $\mathrm{Li}$ et al. [2004a, 2006]. Subsequently, the bundle adjustment (BA) technique was used to build an image network containing all panoramic and traversing images (along with any available VO results) to achieve a highly accurate localization of rover positions along the entire traverse. BA was conducted on the ground (Earth). The strategy and technical details of VO and BA technologies applied at the Meridiani site are similar to those used at the Gusev site and are described by Li et al. [2006].

[19] Visual Odometry is the only technology that gives an MER vehicle a near-real time measurement of how much it slips during a drive. During the first 394 sols on Opportunity, Visual Odometry converged to a solution 95\% (828/ $875)$ of the time. In 111 forward arc drives, the mean commanded distance is $0.45 \mathrm{~m}$, while mean position change derived from $\mathrm{VO}$ is $0.12 \mathrm{~m}$, demonstrating a mean radial slippage of $27 \%$. One example of a successful drive was Sol 304, during which it drove on a bedrock outcrop along the wall of Endurance crater at tilts from 19 to 25 degrees, slipping $10-35 \%$ at each step. Yet Opportunity still reached its target (8.7 meters away) in a single sol, thanks to a clever sequence of commands that adapted driving behavior according to the measured slip.

[20] Figure 7b shows two rover trajectories of Opportunity from Sols 188 to 191, one for wheel odometry (black) and the other for VO (white). The rover was driven uphill and across slope over a total actual distance of $19 \mathrm{~m}$, but wheel odometry underestimated the distance by $1.6 \mathrm{~m}$. The final positions of the wheel-odometry-estimated course and the visual-odometry-corrected course plot differ by nearly
$5 \mathrm{~m}$. Figure $7 \mathrm{a}$ illustrates the trajectories and final positions of the rover for wheel odometry (lower one on the slope) and VO (higher one on the slope) in a simulated Martian surface environment created by JPL software using the DTM, rover model and other data.

[21] Instances where visual odometry failed to converge are primarily attributable to either too large a motion (e.g., commanding a 40-degree turn in place which resulted in too little image overlap) or lack of interesting features for the collection of tie points in the imaged terrain. The plains of Meridiani offer an especially challenging terrain, since the finely grained sand lacks interesting features, but in such terrain we often achieve successful results by imaging rover tracks.

[22] Within Eagle crater, in addition to the set of 3-tier and 360-degree Pancam panoramas, substantial additional Pancam and Navcam images were acquired and used for bundle adjustment. The large number of images within a small crater created a strong image network with a sufficient number of tie points and loops that greatly enhance the BA accuracy. The image network is defined in the LSC system which is further tied to the Mars body-fixed frame by using the localized lander position in the MOC NA imagery (registered in the Mars body-fixed frame) as the origin and IMU observations for the orientation. BA was performed incrementally at each rover location within Eagle crater by fixing the previously adjusted image orientations and only adjusting the newly acquired images at the new locations. The initial values of the new image orientation parameters were extracted/calculated from telemetry data, which include the position and attitude of each rover location derived from IMU and wheel-odometry observations, as well as VO results if performed. As long as the

Figure 8. Opportunity rover traverse comparison up to Sol 62 within Eagle crater. (a) Two-dimensional traverse of the lighter gray dashed line with triangular spots is derived from telemetry data (data transmitted from the rover including wheel encoder and IMU data); 2-D traverse of the dark solid line with circular points is derived from bundle adjustment. (b) Elevation profile comparison. 

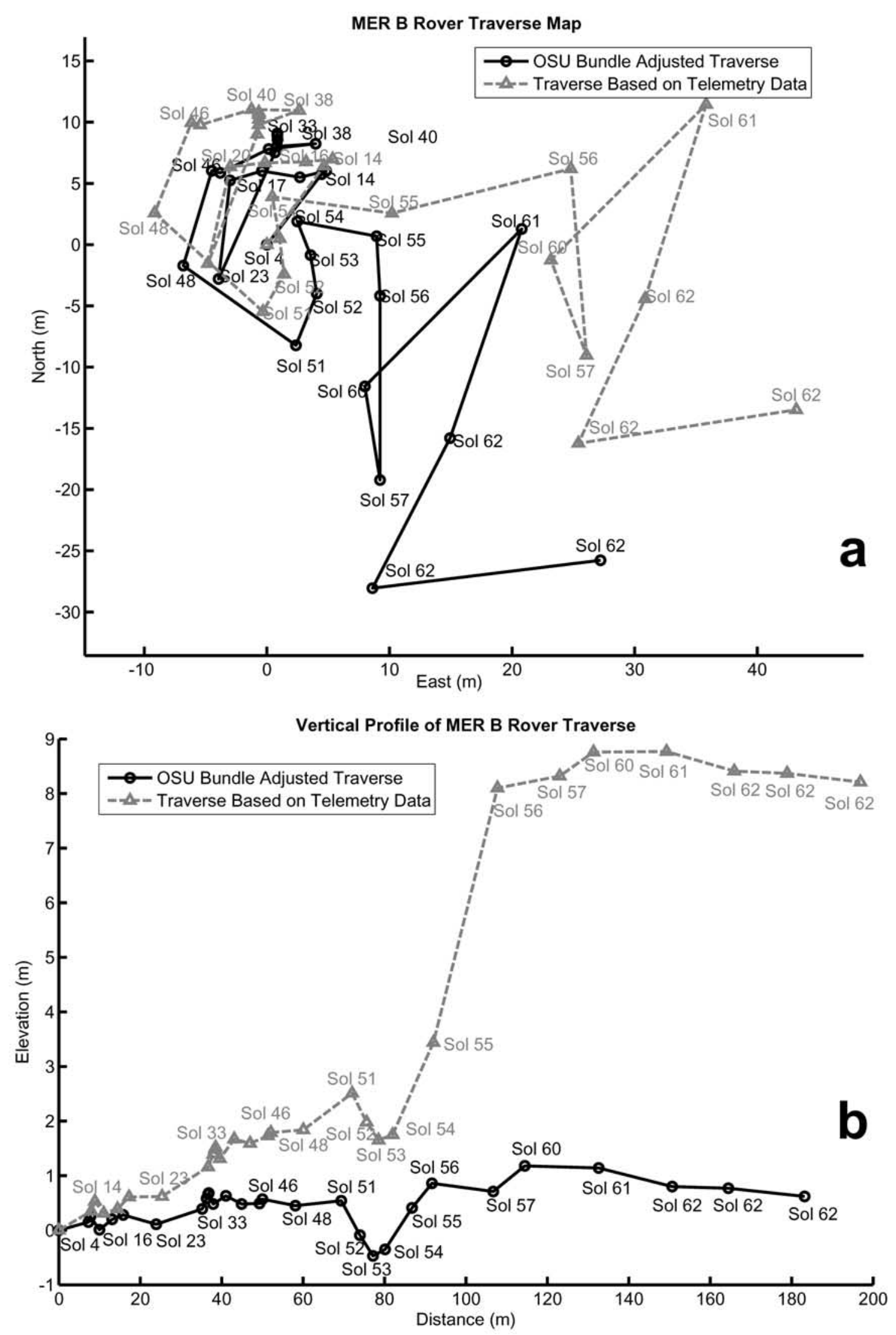

Figure 8 
Oppotunity Rover Traverse (Sol 192)

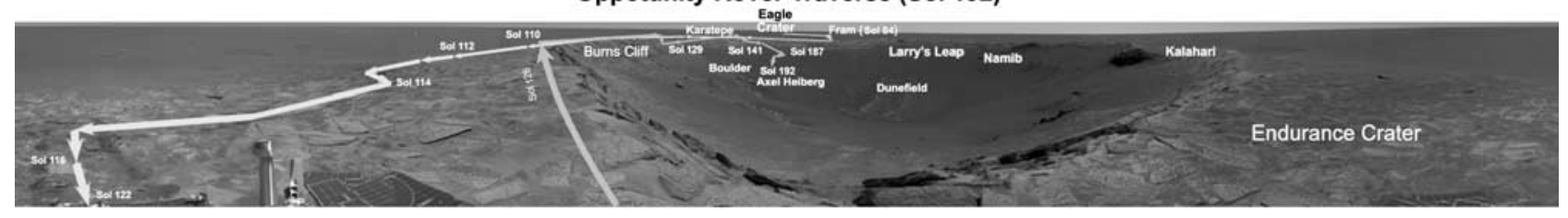

Figure 9. A partial Opportunity rover traverse along and into Endurance crater projected onto a Pancam panorama.

image network is geometrically strong, i.e., a sufficient number of tie points are evenly distributed and are measured accurately, the incremental bundle adjustment provides practically the same results as an integrated bundle adjustment where all the previous images along the traverse are adjusted together. The tie points are mostly located in the overlapping area in the middle between the two rover stations so that the images can be bridged along the traverse. Some of them are on both sides of the traverse, usually within a distance of $20 \mathrm{~m}$ to $30 \mathrm{~m}$ from the rover. This incremental adjustment strategy greatly reduced the computational burden and ensured timely production of rover localization and mapping products for mission operations. The technical details and examples for tie point selection and image network construction are given by Di et al. [2005].

[23] Figure 8a shows the Opportunity rover traverse up to Sol 62 when the rover just exited Eagle crater where the lighter gray dashed line with triangular spots represents the traverse computed using rover telemetry data that are from the rover onboard processing results, including rover positions and attitudes derived from odometry, IMU, and sun imaging data. The onboard VO corrections are also applied whenever performed. On the other hand, the black solid line with circles indicates the traverse in the LSC system through BA. The accumulated difference reached $20.2 \mathrm{~m}$, or $20.5 \%$ of the traveled distance, with a maximum accumulated difference of $21 \mathrm{~m}$ on Sol 62. Significant localization errors in the telemetry data were mainly caused by wheel slippage when Opportunity encountered loose soil on the steep crater wall for 56 sols inside Eagle crater. The BA traverse was able to correct these significant localization errors and put images and observations in context, as well as support science planning and other applications. Furthermore, the BA was performed in three dimensions and there is a $3-\mathrm{D}$ profile along the traverse as a result (Figure $8 \mathrm{~b}$ ). The adjusted profile is in general lower than that derived from telemetry data that are also used to derive the 2-D traverse (Figure 8a), with a maximum difference of $7.63 \mathrm{~m}$. The wheel slippage causes an overestimation of traveled distances and is one of the major reasons for the overestimation of elevations.

[24] Since there is no absolute ground control on the Martian surface, it is impossible to evaluate the accuracy of BA using the conventional method of comparing the bundle adjusted positions with the ground truth. Instead, we estimate the accuracy of the BA by checking consistencies of post-BA positions of the same feature in the 2-D image space and 3-D object space computed from different rover positions along the traverse. Specifically, the 3-D accuracy is derived from the differences of 3-D ground coordinates of the same features triangulated separately from stereo images taken at different rover locations. To calculate the 2-D accuracy, for one ground feature, its coordinates in all images that cover the feature can be back projected and calculated by using the BA results, or directly measured by identifying the features in the images. The 2-D accuracy is
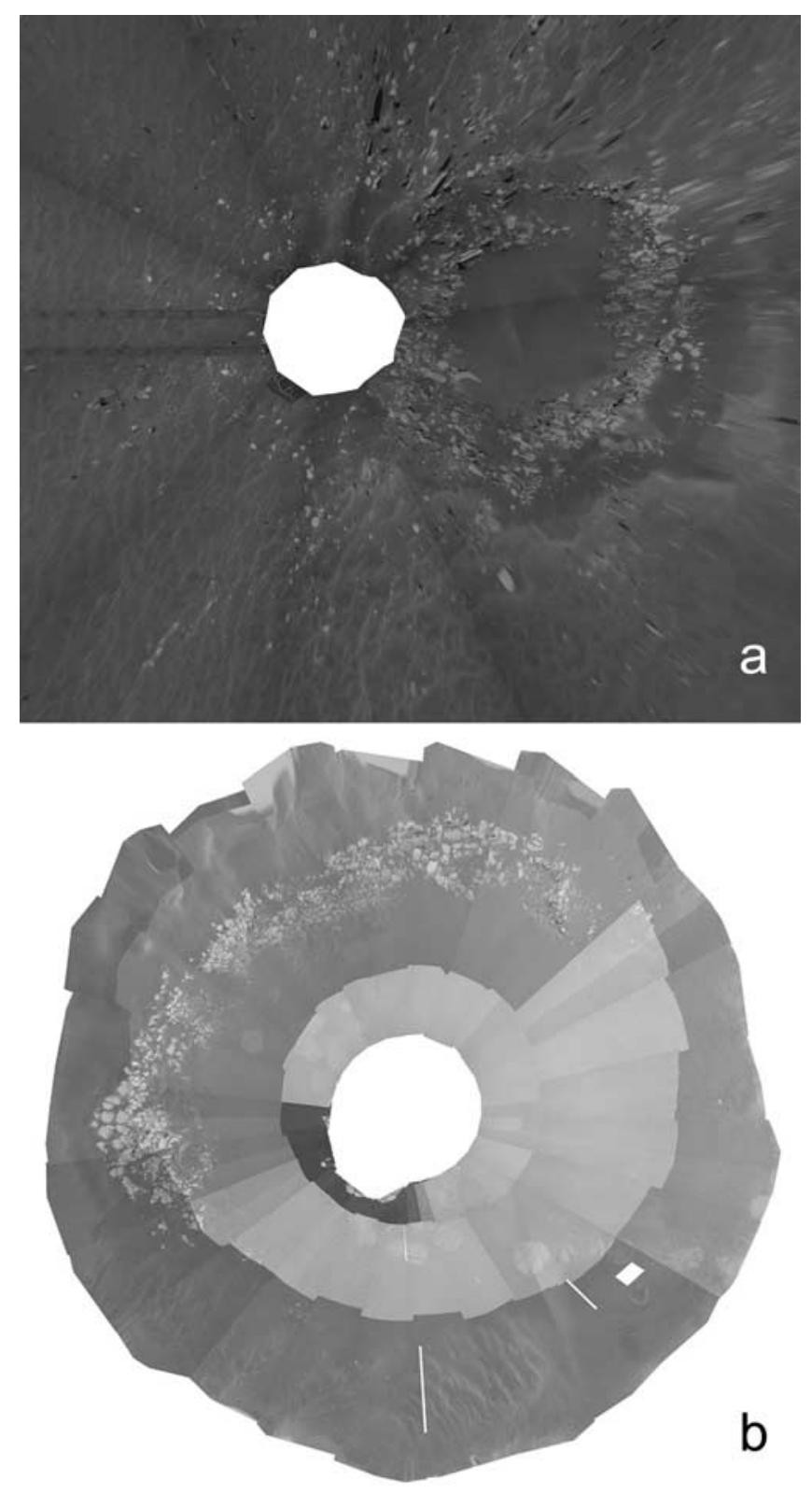

Figure 10. Ortho maps (1 cm/pixel) of (a) Fram crater and (b) Eagle crater. 


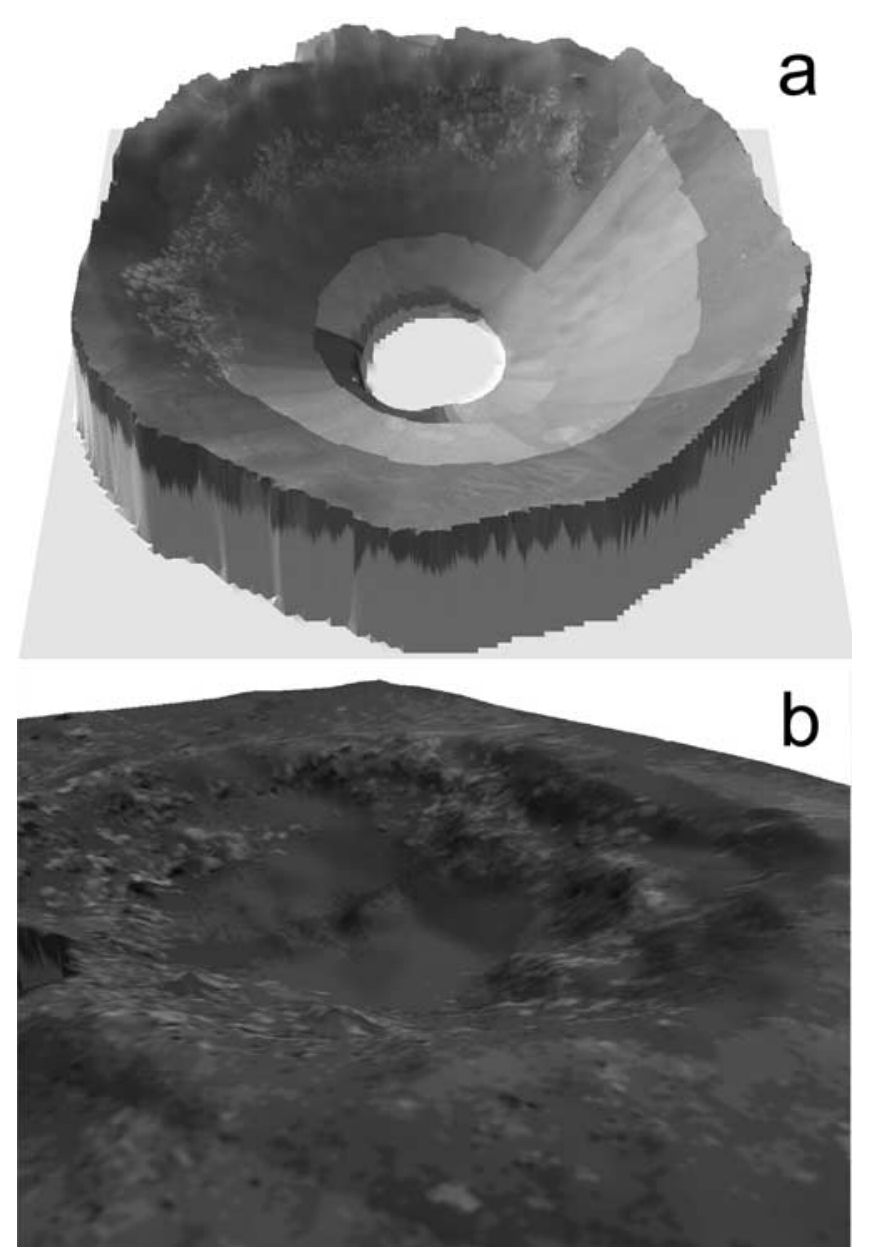

Figure 11. Three-dimensional crater models draped with ortho maps of (a) Eagle crater and (b) Fram crater.

then estimated by using the differences between back projected and measured image coordinates of the same features in two or more stereo images. Within Eagle crater, the 2-D accuracy of BA is typically subpixel and the 3-D accuracy is 2 to $15 \mathrm{~cm}$, depending on the distance from the rover. Here we give an example of bundle adjustment involving images obtained at Positions 200 (Sol 1) and 322 (Sol 16). Fifty-four Pancam images were linked by 665 tie points; before BA, the 2-D errors amounted to more than 100 pixels and 3-D accuracy was $1.493 \mathrm{~m}$. After BA, 2-D accuracy is 0.78 pixel and 3-D accuracy is $0.036 \mathrm{~m}$.

[25] Between Eagle crater and the Anatolia region, there is a data gap for a distance of about $100 \mathrm{~m}$ where the terrain is very flat and no distinguishing land features can be found to bridge the gap. The vast extent of this part of Meridiani Planum is covered by almost repetitive dark grain ripples. The combination of the difficulties made a bundle-adjustmentbased traverse impossible. To continue tracking the rover's global positions, new traverse segments after the gap were computed from telemetry data without BA. These were connected to the end of the bundle-adjusted traverse. By doing so, large features such as the Fram and Endurance craters, when measured from the ground images along the traverse, were generally well matched with their positions on the MOC NA mosaic. This indicates that the rover did not experience significant slippage after exiting Eagle crater, as it had within Eagle crater. However, with Opportunity's busy activities on the crater wall and near the bottom in the dune field inside Endurance crater, significant slippages again occurred. An orthoimage-based traverse adjustment was used to correct these errors. An orthoimage of the entire Endurance crater was generated using two Pancam panoramas taken at the crater rim (Figure 13c). This orthoimage was used as a base map for this adjustment. After the rover entered the crater, comparisons were made between local features in individual orthoimages (made using telemetry data at each rover location) and those in the base map of the global orthoimage. The matched features allowed us to correct the traverse in an effective way. This adjustment method enabled us to provide the Opportunity traverse in a timely and effective manner.

[26] Along the long traverse from Endurance crater southward toward Victoria crater, there were many long drives (e.g., over $150 \mathrm{~m}$ ) that made BA again impossible. Feature comparisons between orbital and rover mages were applied to correct rover location errors. Local orthoimages of craters such as Argo, Jason, Naturaliste and Vostok were generated from rover panoramic images and compared with the MOC NA base map. This orbital-ground comparison proved to be a practical method for rover localization at the Meridiani Planum site. The entire rover traverse as of Sol 458 (Figure 1) is generated using BA, ground-based orthoimage comparisons and orbital-ground comparisons.

[27] We also produced traverse image maps by backprojection of rover positions onto the image mosaics. Figure 9 depicts a partial Opportunity traverse (up to Sol 192) along and into Endurance crater.

\section{High-Resolution Topographic Mapping: Orthoimages and Crater DTMs}

[28] Pancam and Navcam panoramas were often taken at the end of a drive where a new site frame was established. Using these panoramic images, we generated orthoimages

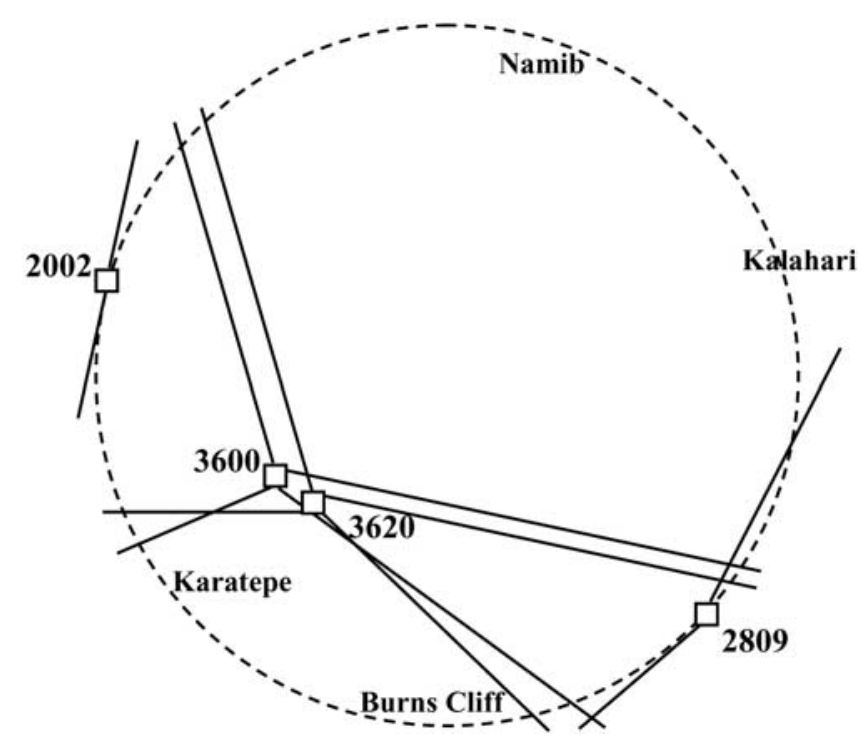

Figure 12. Distribution of panoramic sites for mapping of Endurance crater. 

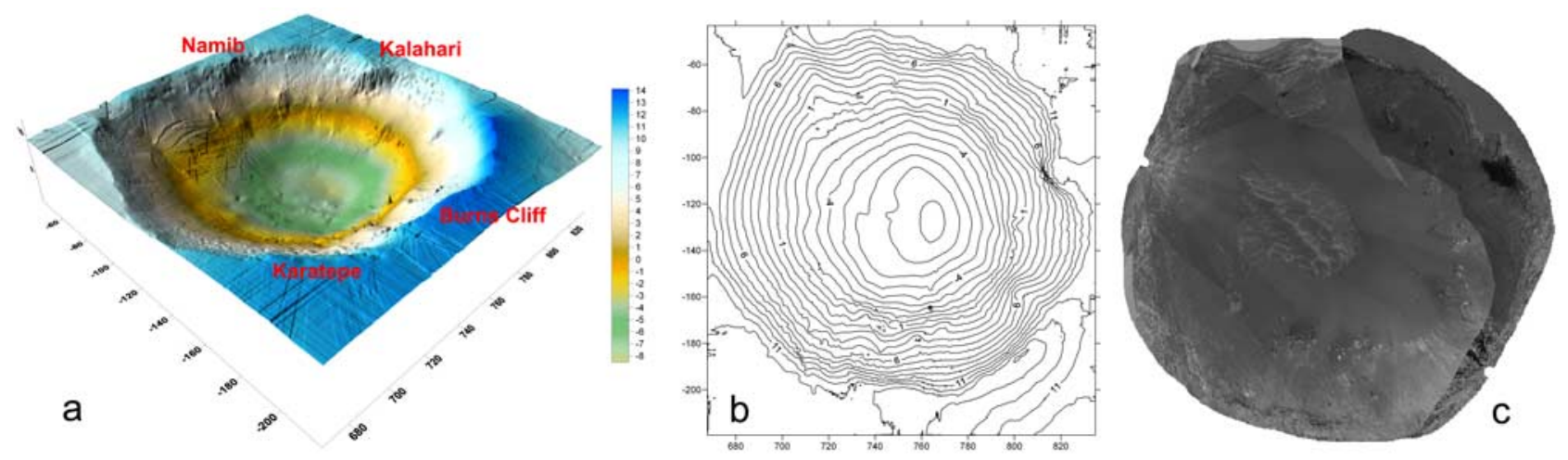

Figure 13. Endurance crater: (a) perspective view of 3-D DTM, (b) contour map, (c) and orthoimage. Ticks on the axes are in meters. Figure 13a has a height exaggeration factor of two.

with $1 \mathrm{~cm}$ resolution. Detailed land features with correct location, orientation, and scale are presented in the orthoimages. These orthoimages are relatively simple to produce when dealing with small craters and hollows such as Fram crater (7 $\mathrm{m}$ in diameter, Figure 10a). However, substantial data processing is required for mapping a large crater where a large data set with panoramas taken at different locations is employed. In such cases, a BA is performed, as described in the following section, to provide a uniform geometric framework. Images with different pointing angles and scales contribute to the overall seamless orthoimage, such as the ones for Eagle crater (22 meters in diameter, Figure 10b) and Endurance crater (156 $\mathrm{m}$ in diameter, Figure 13c).

[29] Craters are the most significant topographical features at the Opportunity site. The Opportunity rover spent extensive time exploring craters and collected stereo Pancam and Navcam images that can be used for crater mapping, for example, at the Eagle, Fram, Endurance and other craters. Opportunity landed inside Eagle crater, the very first rover position is perfect for mapping the crater using the 3 tier and 360 degree Pancam panorama that was also used for triangulating the lander location as mentioned previously. Since the images were acquired at the same location, the BA and subsequent DTM (digital terrain model) and orthoimage generation were performed mostly automatically. Figure 11a shows the digital terrain model of Eagle crater draped with its orthoimage. On the other hand, Fram crater is small. A partial Navcam panorama taken on the crater rim was able to cover the crater and build the DTM (Figure 11b).

[30] Endurance crater is the largest among the craters explored within the operational period covered in this paper. Opportunity spent over 182 sols around and inside the crater, whose diameter is about $156 \mathrm{~m}$ and depth is around $20 \mathrm{~m}$. Two Pancam panoramas (color, three tiers) were taken on the rim: one on Sol 97 (west, Site 2002, 48 stereo pairs) and the other on Sol 122 (southeast, Site 2809, 81 stereo pairs) (Figure 12). The Site ID (e.g., Site 2002) used in this paper is a combination of the rover site and drive position that we use to identify the image or panorama position. These images cover the entire crater. However, on the basis of the stereo vision capability of Pancam cameras, range measurements beyond $80 \mathrm{~m}$ carry great uncertainties. In order to map the entire crater, particularly the region of Namib and the steep crater wall slopes in Karatepe and
Burns Cliff, two more Pancam panoramas were taken, each at Site 3600 and Site 3620 inside Endurance crater. These two panoramas are $5 \mathrm{~m}$ apart and form so-called "wide baseline" stereo images, which increased the stereo baseline from the $30 \mathrm{~cm}$ hard base of the rover to a $5 \mathrm{~m}$ soft base. The increased wide baseline improves the photogrammetric measurement capability and allows topographic mapping of crater features beyond the fixed-range capability.

[31] Among the images collected for mapping Endurance crater, Site 2002 provides details of the west side of the crater; Site 2809 describes the southeast region better; and Sites 3600 and 3620 provide enhanced wide baseline stereo capability to cover the crater bottom and the northern and southern crater walls. The combination of the images from these four sites created an image network that was then bundle adjusted photogrammetrically. Without BA, the uncertainty of features measured in the image space was as large as 414 pixels and $17 \mathrm{~m}$ in the object space. The bundle adjustment of all the images used over 3000 tie points and achieved an accuracy of 0.73 pixels in the image space and $1.9 \mathrm{~m}$ in the object space.

[32] The BA resulted in high precision pointing data of each image in the network and rover positions at the time of imaging, which were then used to compute the accurate 3-D positions of 3-D ground points. Finding corresponding points in stereo or multiple images is a key step in the generation of a large number of 3-D ground points for a DTM. It is mostly carried out automatically by image matching when the images are acquired with a short baseline, for instance, $20 \mathrm{~cm}$ between two Navcam cameras, and correlation between the two images is strong. Although proving improved ranging capability, wide baseline stereo images are less correlated and in general are difficult to handle in image matching. In many cases manual measurements are necessary because of less correlated images. For the Endurance crater model, about 2600 points were generated from wide baseline stereo images at sites 3600 and 3620 , including 2400 automatic points and 200 manual points. In addition, about 98000 points were generated from the "hard" base images at sites 2002 and 2809, of which 500 points are manual points. Since the images are all bundle adjusted, these 3-D points computed from the different sites can be combined to produce a $30 \mathrm{~cm}$ resolution crater DTM by interpolation using the Kriging method. Figure 13a shows a perspective view of the 3 -D crater model and Figure 13b 
the contour map with $1 \mathrm{~m}$ interval. Tics on the axes are in meter. Figure 13a has a height exaggeration factor of two. Using the crater DTM and bundle-adjusted pointing data, we generated three orthoimages covering different regions of the crater. A geometrically seamless mosaic was produced by combining two of the orthoimages for the entire crater (Figure 13c).

\section{Conclusions}

[33] This paper summarizes the achievements of localization and topographic mapping efforts of the MER mission (Opportunity rover) up to Sol 458. Like Spirit rover operations, successful localization of opportunity lander within seven sols after landing and verification by MOC NA orbital images supported science planning in the early stages of surface operations. This is especially important as the spacecraft landed in a crater. Visual odometry has been used frequently to give accurate rover locations when driving toward a target, and has demonstrated its ability to detect the significant slippage that the rover has experienced in Eagle crater and Endurance crater. A ground image network was built by incremental bundle adjustment for the rover traverse within Eagle crater to reduce accumulated rover position errors. The results of visual odometry, bundle adjustments, and orbital-to-ground image-based adjustment ensured that the topographic mapping products of the landing site were generated with high quality for use in strategic planning, tactical operations and many science applications.

[34] Acknowledgments. This work was partially performed at the Jet Propulsion Laboratory, California Institute of Technology, under a contract with the National Aeronautics and Space Administration. Funding of this research by the Mars Exploration Program of NASA is acknowledged. Reviewers' comments are gratefully acknowledged.

\section{References}

Ali, K. S., C. A. Vanelli, J. J. Biesiadecki, M. W. Maimone, Y. Cheng, M. S. Martin, and J. W. Alexander (2005), Attitude and position estimation on the Mars Exploration Rovers, paper presented at IEEE Conference on Systems, Man and Cybernetics, Inst. of Electr. and Electron. Eng., The Big Island, Hawaii, 10-12 Oct.

Arvidson, R. E., et al. (2004), Localization and physical properties experiments conducted by Opportunity at Meridiani Planum, Science, 306(5702), 1730-1733, doi:10.1126/science.1104211.

Bell, J. F., III, J. Joseph, J. N. Sohl-Dickstein, H. M. Arneson, M. J. Johnson, M. T. Lemmon, and D. Savransky (2006), In-flight calibration and performance of the Mars Exploration Rover Panoramic Camera (Pancam) instruments, J. Geophys. Res., 111, E02S03, doi:10.1029/ 2005JE002444.

Biesiadecki, J. J., and M. W. Maimone (2006), The Mars Exploration Rover surface mobility flight software: Driving ambition, paper presented at 2006 IEEE Aerospace Conference, Inst. of Electr. and Electron. Eng., Big Sky, Mont., 4-11 March.

Biesiadecki, J. J., et al. (2005a), Mars Exploration Rover surface operations: Driving Opportunity at Meridiani Planum, paper presented at IEEE
Conference on Systems, Man and Cybernetics, Inst. of Electr. and Electron. Eng., The Big Island, Hawaii, 10-12 Oct.

Biesiadecki, J. J., C. Leger, and M. W. Maimone (2005b), Tradeoffs between Directed and Autonomous Driving on the Mars Exploration Rovers, paper presented at 12th International Symposium on Robotics Research, Int Found. of Robotics Res., San Francisco, Calif., 12-15 Oct.

Cheng, Y., M. W. Maimone, and L. H. Matthies (2005), Visual odometry on the Mars Exploration Rovers, paper presented at IEEE Conference on Systems, Man and Cybernetics, Inst. of Electr. and Electron. Eng., The Big Island, Hawaii, 10-12 Oct.

Cheng, Y., M. W. Maimone, and L. H. Matthies (2006), Visual odometry on the Mars Exploration Rovers, IEEE Robotics Autom., 13(2), spec. issue, $54-62$.

Di, K., F. Xu, J. Wang, X. Niu, C. Serafy, F. Zhou, R. Li, and L. Matthies (2005), Surface imagery based mapping and rover localization for the 2003 Mars Exploration Rover Mission, paper presented at ASPRS 2005 Annual Conference, Am. Soc. for Photogramm. and Remote Sens., Baltimore, Md., 7-11 March.

Golombek, M., and T. Parker (2004), Lander localization, MER Project Memorandum (one for Spirit, one for Opportunity), Jet Propul. Lab., NASA, Pasadena, Calif.

Guinn, J. R., and T. A. Ely (2004), Preliminary results of Mars Exploration Rover in situ radio navigation, paper presented at 14th AAS/AIAA Space Flight Mechanics Meeting, Am. Astron. Soc., Maui, Hawaii, 8-12 Feb.

Kirk, R. L., E. Howington-Kraus, B. Redding, D. Galuszka, T. M. Hare, B. A. Archinal, L. A. Soderblom, and J. M. Barrett (2003), Highresolution topomapping of candidate MER landing sites with Mars Orbiter Camera narrow-angle images, J. Geophys. Res., 108(E12), 8088, doi:10.1029/2003JE002131.

Li, R., K. Di, L. H. Matthies, R. E. Arvidson, W. M. Folkner, and B. A. Archinal (2004a), Rover localization and landing site mapping technology for 2003 Mars Exploration Rover mission, Photogramm. Eng. Remote Sens., 70(1), 77-90.

Li, R., et al. (2004b), Topographic mapping and rover localization in MER 2003 mission landing sites, Eos Trans. AGU, 85(17), AGU Jt. Assem. Suppl., Abstract P33D-17.

$\mathrm{Li}$, R., et al. (2006), Spirit rover localization and topographic mapping at the landing site of Gusev crater, Mars, J. Geophys. Res., 111, E02S06, doi:10.1029/2005JE002483.

Malin, M. (2004), MOC cPROTO and ROTO images with Spirit and Opportunity landers, Malin Space Sci. Syst., San Diego, Calif. (Available at http://www.msss.com/mer_mission/index.html)

Olson, C. F., L. H. Matthies, M. Schoppers, and M. W. Maimone (2003), Rover navigation using stereo ego-motion, Robotics Auton. Syst., 43(4), $215-229$.

Parker, T., et al. (2004), Localization, localization, localization, Lunar Planet. Sci., XXXV, Abstract 2189.

Squyres, S. W., et al. (2004), In situ evidence for an ancient aqueous environment at Meridiani Planum, Mars, Science, 306(5702), 17091714, doi:10.1126/science.1104559.

R. E. Arvidson, Department of Earth and Planetary Sciences, Washington University, St. Louis, MO 63130, USA.

K. Di and R. Li, Department of Civil and Environmental Engineering and Geodetic Science, Ohio State University, Columbus, OH 43210, USA. (li.282@osu.edu)

M. Golombek, J. Guinn, A. Johnson, M. Maimone, L. H. Matthies, and T. Parker, Jet Propulsion Laboratory, California Institute of Technology, Pasadena, CA 91125, USA.

M. Malin, Malin Space Science Systems, San Diego, CA 92191, USA.

S. W. Squyres, Department of Astronomy, Cornell University, 428 Space Sciences Building, Ithaca, NY 14853, USA.

W. A. Watters, Department of Earth, Atmospheric and Planetary Sciences, Massachusetts Institute of Technology, 77 Massachusetts Avenue, Cambridge, MA 02139, USA. 